Research Note

\title{
Multimode interference-based fiber sensor in a cavity ring-down system for refractive index measurement
}

\author{
Susana Silva*, Orlando Frazão \\ INESC TEC and Department of Physics and Astronomy, Faculty of Sciences of University of Porto, Rua do Campo Alegre 687, 4169-007 Porto, Portugal
}

\section{A R T I C L E I N F O}

\section{Keywords:}

Cavity ring down

Refractive index

Multimode interference

Optical fiber sensors

\begin{abstract}
A B S T R A C T
This work reports a multimode interference-based fiber sensor in a cavity ring-down system (CRD) for sensing temperature-induced refractive index (RI) changes of water. The sensing head is based in multimodal interference (MMI) and it is placed inside the fiber loop cavity of the CRD system. A modulated laser source was used to send pulses down into the fiber loop cavity and an erbium-doped fiber amplifier (EDFA) was placed in the fiber ring to provide an observable signal with a reasonable decay time. The behavior of the sensing head to temperature was studied due to its intrinsic sensitivity to said parameter - a sensitivity of $-1.6 \times 10^{-9} \mu \mathrm{s} /{ }^{\circ} \mathrm{C}$ was attained. This allowed eliminating the temperature component from RI measurement of water and a linear sensitivity of $580 \mu \mathrm{s} / \mathrm{RIU}$ in the RI range of 1.324-1.331 was obtained. The use of a MMI fiber sensor in the proposed CRD configuration allowed achieving a sensitivity $\sim 4$-fold than that obtained with a tilted fiber Bragg grating and $\sim 2$-fold than that when a micrometric channel inscribed in the fiber was used.
\end{abstract}

\section{Introduction}

Cavity ring-down (CRD) spectroscopy is a well-established technique that has been target of research in the spectroscopy field, especially by the demonstration of highly sensitive direct absorption measurements with pulsed light sources [1]. The implementation of optical fibers led to new fiber optic-based CRD systems which, in turn, used a fiber loop operating as the resonant cavity. This configuration quickly obtained popularity in the scientific community, mainly for presenting an effective alternative to the usual CRD configuration [2]. However, CRD systems present an almost $100 \%$ coupling loss when light is coupled into the cavity, either using reflective layers ( $R>99.9 \%$ ) or optical fiber couplers with high splitting ratios (99:1) in the case of fiber loop configurations. To overcome this issue, the used of an erbium-doped fiber amplifier (EDFA) has been reported for loss compensation, increasing the ring-down time of the system [3]. The study of signal amplification by placing an EDFA inside a fiber ring configuration was also reported [4]. Recently, a fiber-based CRD technique that used a large core multimode fiber-cavity design based on highly reflective gold coatings was demonstrated [5]. Recently, it is demonstrated the effect of using an EDFA for signal amplification inside the fiber ring of a cavity ring-down configuration [6].

In the last decade, the CRD technique has been devoted to sensing applications [7]. Many efforts have been made in developing optical fiber sensors suitable for measuring refractive index (RI), when integrated in a CRD configuration. The challenge of this task is to obtain a fiber structure sensitive to the external medium, enough to originate amplitude variation of the optical spectrum and, consequently, inducing losses on the CRD output signal. In early reports, the Long Period Grating (LPG) was found to be a suitable solution for RI sensing in a CRD configuration [8]. The LPG was placed inside the fiber ring and studied the ring-down decay time of the fiber loop as a function of the RI variation of the external medium. Results indicated an amplitude signal decay of $7.16 \mathrm{~dB}$ in the RI range of 1.35-1.43, corresponding to a ring-down time of $27.03 \mu \mathrm{s}$ and a net loss of the CRD loop of $0.69 \mathrm{~dB}$. Later, a tilted fiber Bragg grating was proposed as sensing element inside the fiber loop [9]; using this configuration, a maximum sensitivity of $154 \mu \mathrm{s} / \mathrm{RIU}$ in the high RI range was obtained and a resolution of $6.5 \times 10^{-6}$ RIU. Also, a micrometric channel was inscribed in the fiber of the cavity ring by means of femtosecond technique [10]. This configuration allowed measuring gels with different RIs; a sensitivity up to $300 \mu \mathrm{s} /$ RIU close to the RI of 1.452 was obtained and also a resolution of $1.4 \times 10^{-4}$ RIU. In a different approach, a partially etched cladding singlemode fiber was used as sensing element inside the fiber ring [11]. A maximum detection limit for an RI change of $3.2 \times 10^{-5}$ was demonstrated in the RI range of 1.3388-1.3398. In this range, ring-down times between 8.8 and $9.6 \mu \mathrm{s}$ were also obtained. Recently, a Mach-Zehnder interferometer based on a Photonic Crystal Fiber was integrated in the cavity ring for the detection of RIs in liquid medium [12]. This configuration presented a

\footnotetext{
* Corresponding author

E-mail addresses: sfsilva@inesctec.pt (S. Silva), ofrazao@inesctec.pt (O. Frazão).
} 


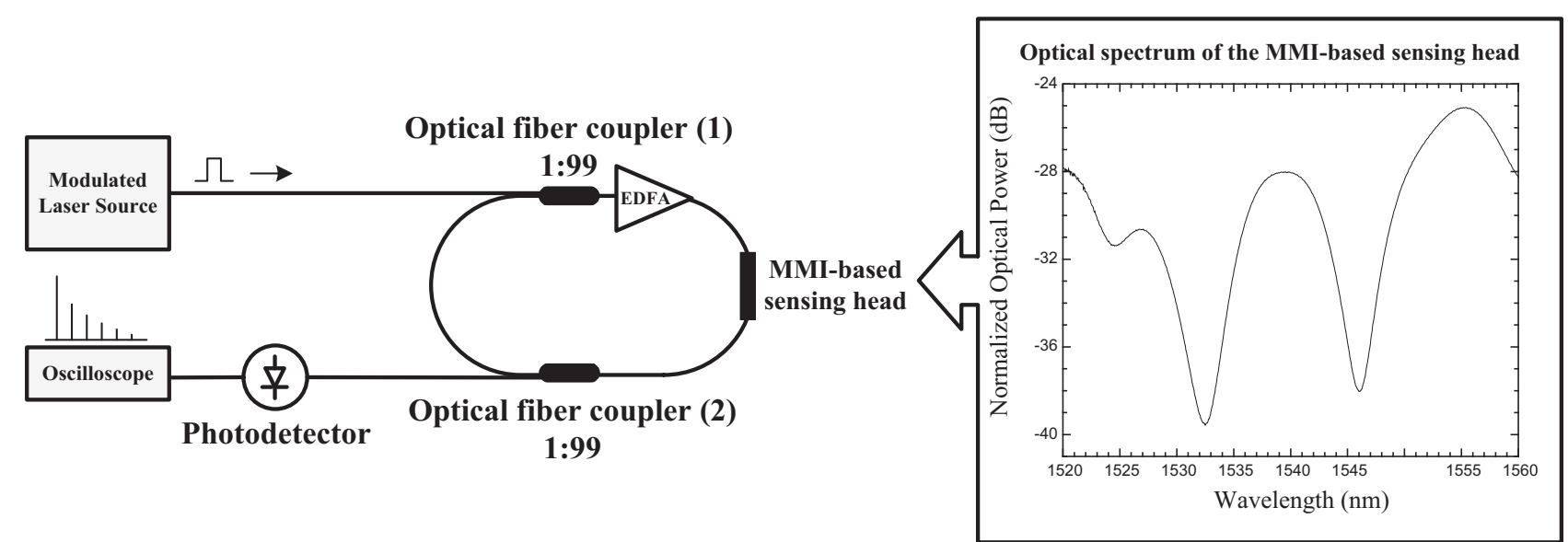

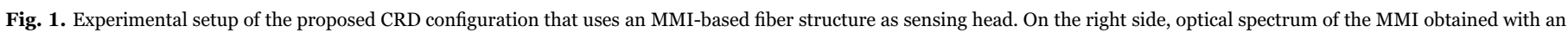
optical spectrum analyzer.

linear sensitivity of $11.7 \mu \mathrm{s}^{-1} \mathrm{RIU}^{-1}$ with a corresponding minimum detectable of $7.8 \times 10^{-5}$ RIU.

This work presents a CRD system that uses an MMI-based fiber device inside the fiber loop for measuring RI. The sensing head is based on multimodal interference, and relies on a singlemode - corelessmultimode - singlemode fiber configuration. One limitation of this type of MMI-based sensing head is that presents high losses; in order to solve the problem, an EDFA was incorporated in the loop cavity. The sensing device was submitted to RI changes of water caused by temperature variation.

\section{Experimental results}

In this approach, the CRD system is composed by a modulated laser source, two standard 1:99 optical fiber couplers $(2 \times 1)$, a fiber loop with $\sim 1 \mathrm{~km}$ (SMF 28), an EDFA, a photodetector, and an oscilloscope. Inside the fiber loop was placed the MMI-based fiber device for sensing purposes. The experimental setup of the proposed CRD configuration is shown in Fig. 1 and, on the right side, the optical spectrum of the MMIbased fiber sensor obtained with an optical spectrum analyzer.

The modulated laser source is used to send pulses (500 ns@ $1550 \mathrm{~nm}$ ) down into the fiber loop - the train of pulses is coupled via $1 \%$ arm of the input optical coupler, rings around inside the fiber loop, and is coupled out via $1 \%$ arm of the output coupler; the amplitude of the output pulses decays with time due to the total existing losses in the fiber loop (fiber loss, fiber couplers insertion losses, MMI transmission attenuation), passes through a photodetector (gain of $40 \mathrm{~dB}$ ), and is monitored in an oscilloscope. The EDFA was made in the lab, has $2 \mathrm{~m}$ of an erbium-doped fiber (losses of $14 \mathrm{~dB} / \mathrm{m} @ 980 \mathrm{~nm}$ ) and it was inserted in the fiber loop for signal amplification of the CRD configuration. In order to compensate the losses from the MMI-based sensing head, a current of $155.7 \mathrm{~mA}$ was applied to the pump-diode of the EDFA.

The sensing head is based on multimodal interference and relies on a singlemode - coreless-multimode - singlemode fiber configuration (SMF - coreless-MMF - SMF). The coreless-MMF is a pure silica fiber with $125 \mu \mathrm{m}$ diameter and $20 \mathrm{~mm}$-length, which was spliced between two SMFs and interrogated in transmission (see Fig. 1b). The principle of this fiber structure underlies on the concept that when the light field coming from the input SMF enters the coreless-MMF, interference between the different modes occurs along the MMF section. The light is coupled into the output SMF - and it will depend on the amplitudes and relative phases of the several modes at the exit end of the corelessMMF. Therefore, the coupling efficiency, for a given length of the MMF section, is strongly wavelength-dependent. In this work, the operating mechanism of the sensing head relies on destructive interference at the output end of the coreless-MMF section, which occurs for a specific length of the MMF used $(20 \mathrm{~mm})$. The result is a band-rejection filter in the operation wavelength range of $1520-1560 \mathrm{~nm}$ (see Fig. 1b). It is expected that the MMI-based fiber sensor head is sensitive to the external medium, namely to RI trough wavelength variation [13]. Thus, the wavelength variation is due to the effective index variation of the guided modes of the coreless fiber-MMF when subjected to RI changes of the external medium. This behavior of the sensing head comes with a variation of the signal amplitude as a consequence of the change in reflection coefficient at the multimode fiber-to-liquid interface. The signal amplitude decreases with increasing RI of the external medium as it approaches the silica RI (coreless-MMF). Thus, the sensing head developed in this work is able to be used as an intensity sensor.

The CRD trace obtained with the proposed configuration is shown in Fig. 2. This waveform has a ring-down time of $11.1 \mu$ s and is strongly dependent on the pulse width, fiber length, fiber losses, MMI losses and others. The time of a single round trip is ca. $7.8 \mu \mathrm{s}$ is determined by the cavity length.

The behavior of the MMI-based fiber sensor as a refractometer was duly characterized. The sensing head was placed under water, fixed on a holder in order to eliminate any bending variations, and submitted to increasing temperature in the range $25-80{ }^{\circ} \mathrm{C}$, with $5{ }^{\circ} \mathrm{C}$ steps. The modulated laser source used in this experiment sent pulses at the operation wavelength of $1550 \mathrm{~nm}$, which in turn is located in the slope of the band-rejection filter centered at $1546 \mathrm{~nm}$ (see Fig. 1b). Therefore, the wavelength variation caused by RI changes of the

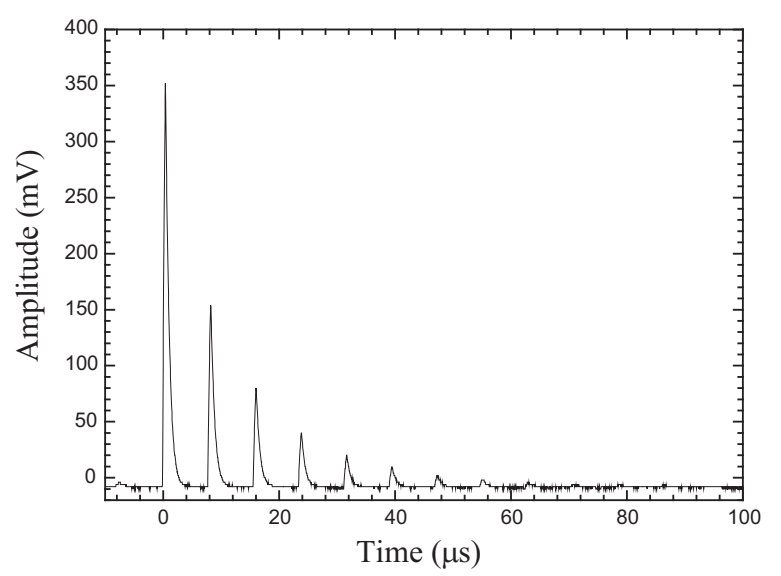

Fig. 2. CRD trace for pulses sent by the modulated multimode laser source into the fiber loop with $500 \mathrm{~ns}$ width. 


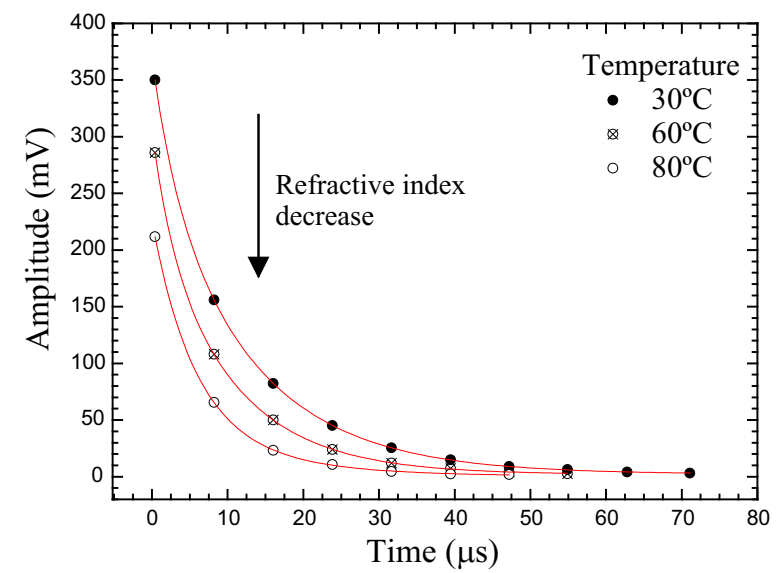

Fig. 3. Exponential fitting of the CRD trace for different introduced losses caused by temperature-induced RI variation of water.
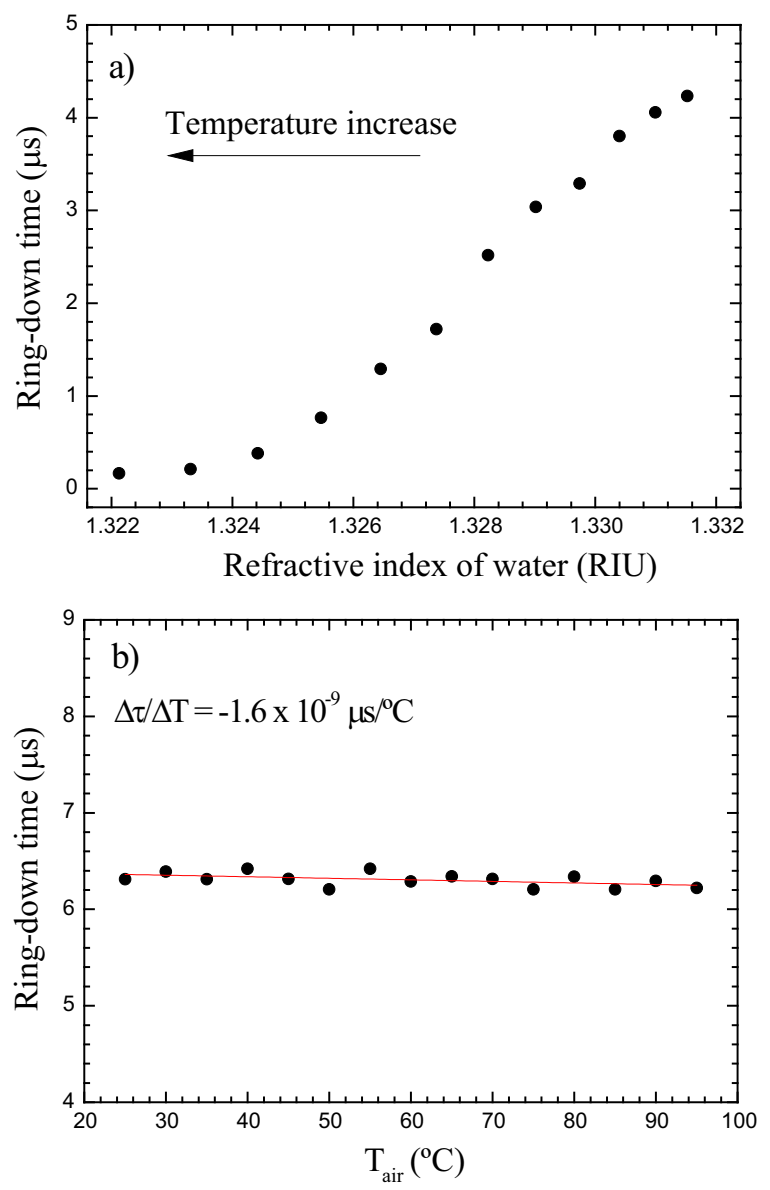

Fig. 4. Ring-down time versus (a) temperature-induced RI changes of water and (b) temperature applied to the MMI-based fiber sensor integrated in the CRD system.

external medium will shift the band-rejection peaks, associated with an amplitude variation of the acquired signal. Fig. 3 shows the exponential fitting of the CRD trace for different introduced losses caused by temperature-induced RI variation of water.

In this case, the temperature variation of water causes the amplitude of the band-rejection peaks to decrease and consequently leading to measurable losses in the acquired CRD signal. The different associated losses led to different ring-down times, as observed in Fig. 4a.
Table 1

RI sensitivity for different sensing head configurations.

\begin{tabular}{lll}
\hline Sensors Type & RI sensitivity & Reference \\
\hline LPG & - & {$[8]$} \\
Tilted fiber Bragg grating & $154 \mu \mathrm{s} / \mathrm{RIU}$ & {$[9]$} \\
Micrometric channel inscribed in the fiber & $300 \mu \mathrm{s} / \mathrm{RIU}$ & {$[10]$} \\
Etched cladding singlemode fiber & - & {$[11]$} \\
Mach-Zehnder interferometer in PCF & $11.7 \mu \mathrm{s}^{-1} \mathrm{RIU}^{-1}$ & {$[12]$} \\
This work & $\mathbf{5 8 0} \mathbf{\mu s} / \mathbf{R I U}$ & - \\
\hline
\end{tabular}

The ring-down time decreases with RI of water as a result of temperature increase, as shown in Fig. 4a. The temperature-RI scale conversion was based on the RI dependence of water with wavelength (in this case, $1550 \mathrm{~nm}$ ), constant pressure and temperature [14]. Since the MMI-based fiber sensor is intrinsically sensitive to temperature but also to RI of the external medium, one had to remove the temperature component of the acquired output signal, in order to obtain the results presented in Fig. 4a.

Therefore, the behavior of the sensing head to temperature variation was studied. The MMI fiber sensor was placed in a furnace and submitted to increasing temperature in the range $25-95{ }^{\circ} \mathrm{C}$, with $5{ }^{\circ} \mathrm{C}$ steps. A negligible sensitivity of $-1.6 \times 10^{-9} \mu \mathrm{s} /{ }^{\circ} \mathrm{C}$ was attained, as depicted in Fig. 4b. This allowed eliminating the temperature component from RI measurement of water and a linear sensitivity of $580 \mu \mathrm{s} /$ RIU in the RI range of 1.324-1.331 was obtained. For comparison purposes, Table 1 lists the RI sensitivity for other sensing head configurations already reported.

\section{Conclusions}

Summarizing, an MMI-based fiber device was inserted in the fiber loop and interrogated by CRD Technique for measuring temperatureinduced RI changes of water. The sensing head was based on multimodal interference, and relied on a (SMF - coreless-MMF - SMF) fiber configuration. Due to the high losses intrinsic of the MMI fiber structure, an EDFA was inserted in the fiber loop cavity in order to compensate them. This allowed obtaining an observable signal with a reasonable decay time. The behavior of the sensing head to temperature variation was also studied and a sensitivity of $-1.6 \times 10^{-9} \mu \mathrm{s} /{ }^{\circ} \mathrm{C}$ was attained. This allowed eliminating the temperature component from RI measurement of water and a linear sensitivity of $580 \mu \mathrm{s} / \mathrm{RIU}$ in the RI range of 1.324-1.331 was obtained. The MMI fiber sensor allowed achieving a sensitivity $\sim$-fold than that already reported with a tilted fiber Bragg grating and $\sim 2$ fold than that when a micrometric channel inscribed in the fiber was used.

\section{Acknowledgments}

This work was supported by Project CORAL - Sustainable Ocean Exploitation: Tools and Sensors, NORTE-01-0145-FEDER-000036, financed by the North Portugal Regional Operational Programme (NORTE 2020), under the PORTUGAL 2020 Partnership Agreement, and through the European Regional Development Fund (ERDF). S.S. received a Pos-Doc fellowship (ref. SFRH/BPD/92418/2013) also funded by FCT - Portuguese national funding agency for science, research and technology.

\section{References}

[1] A. O'Keefe, D.A.G. Deacon, Cavity-ring-down optical spectrometer for absorption measurements using pulsed laser sources, Rev. Sci. Instrum. 59 (1988) 2544-2551.

[2] G. Stewart, K. Atherton, B. Culshaw, Cavity-enhanced spectroscopy in fiber cavities, Opt. Lett. 29 (5) (2004) 442-444.

[3] G. Stewart, K. Atherton, H. Yu, B. Culshaw, An investigation of an optical fiber amplifier loop for intra-cavity and ring-down cavity loss measurements, Meas. Sci. Technol. 12 (12) (2001) 843-849. 
[4] B. Vizoso, C. Vfizquez, R. Civera, M. Lopez-Amo, M.A. Muriel, Amplified fiber-optic recirculating delay lines, J. Lightwave Technol. 12 (2) (1994) 294-305.

[5] M. Fabian, E. Lewis, T. Newe, S. Lochmann, Optical fiber cavity for ring-down experiments with low coupling losses, Meas. Sci. Technol. 21 (9) (2010) 094034.

[6] S. Silva, R. Magalhães, R.A. Pérez-Herrera, M. Lopez-Amo, M.B. Marques, O. Frazão, Fiber cavity ring down and gain amplification effect, Photonic Sens. 4 (6) (2016) 324-327.

[7] S.O. Silva, R. Magalhães, M.B. Marques, O. Frazão, New advances in fiber cavity ring-down technology, Opt. Laser Technol. 78 (2016) 115-119.

[8] N. Ni, C. Chan, L. Xia, P. Shum, Fiber cavity ring-down refractive index sensor, IEEE Photonics Technol. Lett. 20 (16) (2008) 1351-1353.

[9] K.M. Zhou, D. Webb, M. Farries, N. Hayes, L. Zhang, I. Bennion, Biochemical sensor based on a novel all-fibre cavity ring down spectroscopy technique incorporating a tilted fibre Bragg grating, Opt. Lasers Eng. 47 (2009) 1023-1027. [10] K.M. Zhou, D.J. Webb, C.B. Mou, M. Farries, N. Hayes, I. Bennion, Optical fiber cavity ring down measurement of refractive index with a microchannel drilled by femtosecond laser, IEEE Photonics Technol. Lett. 21 (22) (2009) 1653-1655.

[11] C. Wang, C. Herath, High-sensitivity fiber-loop ringdown refractive index sensors using single-mode fiber, Opt. Lett. 35 (10) (2010) 1629-1631.

[12] W.C. Wong, W. Zhou, C.C. Chan, X. Dong, K.C. Leong, Cavity ringdown refractive index sensor using photonic crystal fiber interferometer, Sens. Actuators B 161 (2012) 108-113.

[13] O. Frazão, S. Silva, J. Viegas, L.A. Ferreira, F.M. Araújo, J.L. Santos, Optical fiber refractometry based on multimode interference, Appl. Opt. 50 (2011) E184-E188.

[14] International Critical Tables of Numerical Data, Physics, Chemistry and Technology, McGraw-Hill Book Company, New York and London, 1930. 